\title{
The Partially-Coherent AWGN Channel: Transceiver Strategies for Low-Complexity Fibre Links
}

\author{
Hubert Dzieciol, Student Member, IEEE, Eric Sillekens, Member, IEEE, Gabriele Liga, Member, IEEE, \\ Polina Bayvel, Fellow, IEEE, Robert Killey, Senior Member, IEEE, and Domaniç Lavery, Member, IEEE
}

\begin{abstract}
Carrier phase estimation (CPE) is one of the key requirements to perform intradyne coherent detection in optical communication systems. Residual errors in the phase estimation at the receiver, also known as residual phase noise (RPN), follow the so-called Tikhonov distribution. In the digital domain, a channel where the phase has already been estimated by the CPE is generally known as a partially-coherent additive white Gaussian noise (PCAWGN) channel. Herein, we present a joint strategy to modulate and demodulate a 2-dimensional (2D) signal in a PCAWGN channel. Using a low-complexity demapper, we geometrically shape (GS) 8- to 64-ary modulation formats for a PCAWGN channel. Through numerical simulations, we then assess the bit-wise achievable information rates (AIRs) and post forward error correction (FEC) bit error rates (BER) of the presented constellations with the: theoretical optimum model, Euclidean model and the low-complexity PCAWGN model. The resulting constellations are shown to be tolerant to a significant amount of RPN and are therefore applicable to coherent optical communication systems using high linewidth lasers (e.g., > $500 \mathrm{kHz}$ ) and/or lower symbol rates. Moreover, we demonstrate that shaped PCAWGN constellations combined with a low-complexity demapper can either significantly relax laser linewidth (LW) or carrier phase estimation (CPE) requirements. Assuming a rate-9/10 LDPC scheme, we demonstrate post-FEC BER shaping gains of up to $2.59 \mathrm{~dB}$ and $2.19 \mathrm{~dB}$ versus uniform 64QAM and 64-ary constellations shaped for the purely AWGN channel, respectively.
\end{abstract}

Index Terms-Phase noise, Signal design, Phase estimation, Optical fiber communication

\section{INTRODUCTION}

In coherent optical fibre communications, information can be encoded in both the amplitude and phase of the signal. The range of linear and non-linear impairments observed in

Manuscript received July, 2020. This work was funded by the United Kingdom (UK) Engineering and Physical Sciences Research Council (EPSRC) Programme Grant TRANSNET (Transforming networks - building an intelligent optical infrastructure, EP/R035342/1). H. Dzieciol is in receipt of a $\mathrm{PhD}$ studentship from the EPSRC and Microsoft Research. The work of G. Liga is funded by the EUROTECH postdoc programme under the European Union's Horizon 2020 research and innovation programme (Marie Skłodowska-Curie grant agreement No 754462). D. Lavery is supported by the Royal Academy of Engineering under the Research Fellowships scheme.

H. Dzieciol, E. Sillekens P. Bayvel and R. Killey are with the Optical Networks Group, Department of Electronic and Electrical Engineering, University College London, London WC1E 7JE, U.K. (e-mail: \{hubert.dzieciol.18, e.sillekens, p.bayvel and r.killey\}@ucl.ac.uk).

G. Liga is with the Signal Processing Systems (SPS) Group, Department of Electrical Engineering, Eindhoven University of Technology, The Netherlands. (e-mail: g.liga@tue.nl).

D. Lavery was with the Optical Networks Group, Department of Electronic and Electrical Engineering, University College London, London WC1E 7JE, U.K., and is now with Infinera Canada Inc., Ottawa, ON, Canada. (e-mail: dlavery@ieee.org). these systems is typically modelled as additive white Gaussian noise (AWGN). Moreover, the finite linewidths (LWs) of the source and local oscillator (LO) lasers [1] introduce the additional phase noise component into the signal. If the LWs are sufficiently low, the phase noise impact can be compensated by carrier phase estimation (CPE) algorithms, and thus neglected by the symbol demapper. However, in scenarios in which the LWs are relatively high, and the CPE fails to fully track the incoming phase noise, doing so may result in significant signal-to-noise ratio (SNR) penalties. These penalties are due to residual phase fluctuation in the recovered signal constellation and are commonly referred to as the residual phase noise (RPN).

Typically, the phase error estimation comes after the other signal conditioning subsystems such as filtering or equalisation and is performed via digital signal processing (DSP) [2]. The output of the CPE is impaired only with AWGN and a portion of the angular phase noise (i.e. RPN or a phase 'jitter') that the algorithm failed to estimate. Such a channel is generally known as a partially coherent AWGN (PCAWGN) model and has been extensively studied in the past [3]-[6].

In the area of optical communications, most of the work related to signal shaping focuses on AWGN auxiliary channels (i.e. the channels often approximated by an equivalent of AWGN channel) [7]-[12]. However, when dealing with lowcomplexity transmission systems, where: the laser LWs are generally high $(>500 \mathrm{kHz})$ [13] and/or the baud rate is extremely low ( $<10$ Gbaud) [14], the impact of the RPN may significantly increase the SNR requirements of the link. In such scenarios, it is worth taking the increased amount of RPN into account for the transmitter (TX) design optimisation. Alternatively, it is also possible to improve the CPE; however, this approach may either reduce the net information rate of the link or add additional complexity due to a more sophisticated CPE strategy.

Since Foschini's seminal paper in 1973 [3], several studies addressed the properties of the PCAWGN channel [5]. In the context of signal design, the works in [15] and [16] demonstrated optimisation techniques for 2-dimensional (2D) modulation formats that focused on maximising the performance of non-binary coding schemes. It was followed by [6], where the authors used a simulated annealing algorithm [17] to geometrically shape a range of 8- to 256-ary constellations for both symbol-wise and bit-wise (BW) achievable information rates (AIRs) [18]. However, due to computational limitations, a 2-fold symmetry condition was imposed on the higher order modulation formats. Furthermore, the studies in [19] 
(a)

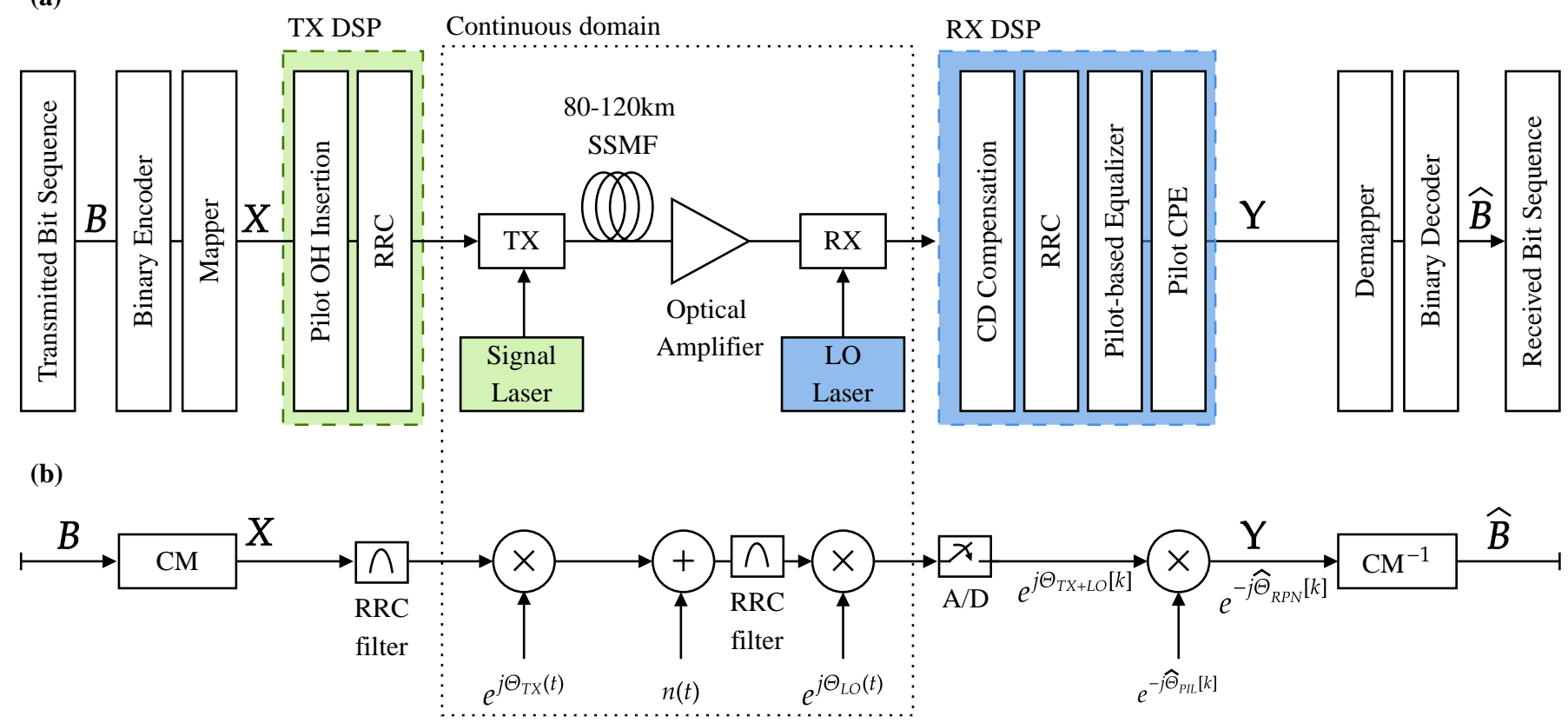

Fig. 1. A system model (a) and the equivalent mathematical representation (b), of a typical short-range optical fibre communication link employing coded modulation (CM) with soft decision FEC. It is impaired by chromatic dispersion (CD) which is compensated at the receiver. The term $n(t)$ stands for AWGN process with power spectral density of $\mathcal{N}_{o}$ and the analogue-to-digital conversion is denoted as A/D.

and [20] proposed heuristic design methods to increase the constellation's robustness in a phase noise dominated channel. Alternatively, the work in [21] demonstrated significant reach gains by using the PCAWGN model in non-linear channel transmission for both probabilistic and geometrically shaped (GS) formats. Lastly, a recent study in [22] looked at employing end-to-end deep learning model to geometrically shape phase noise-robust multi-dimensional constellations for optical communications in PCAWGN channel.

Generally, when compared to the circular Gaussian model [23], the PCAWGN receiver (RX) requires significantly more computational resources to compute the log-likelihood ratios (LLRs) of the received bits that are the fundamental basis of soft-decision (SD) coding schemes. However, recent work in [4] addressed this problem and proposed a low-complexity approximation of the channel that is based on polar coordinates. By transforming the statistical distribution of the phase noise to a truncated version of the unwrapped Gaussian probability density function (PDF), the authors reduced the (estimated) number of mathematical operations required to calculate the conditional probability of a PCAWGN model [4, Table I].

In our previous work [24], we designed a range of shaped modulation formats for the PCAWGN channel, focusing explicitly on channel parameters originating from the specifications given by the 400ZR implementation agreement [13]. The proposed constellations were shaped via a Monte Carlo approach, in which, we maximised the generalized mutual information (GMI) [25], [26] of each symbol arrangement in the presence of both AWGN and high levels of RPN, assuming a typical, circular Gaussian metric LLR calculation. In other words, we tried to minimise the computational overhead required to implement this strategy in 400ZR links. Although substantial performance gains were demonstrated versus square 16-QAM, and also for the higher cardinality formats, it was not determined whether a more accurate, yet low-complexity, model of the RX could further decrease the SNR penalties by offering an increased phase noise tolerance in such a system. Moreover, whether optimizing constellations for such receiver could bring more significant gains.

Herein, we look at the low-complexity PCAWGN demapper proposed in [4] and use it to improve on our previous constellation optimisation algorithm in [24]. Then, we maximise the BW AIRs of the GS 8 to 64-ary constellations in the presence of both AWGN and RPN. In contrast with the work in [6], no symmetry conditions are imposed on the optimiser. In the next stage, we verify the resulting PCAWGN-shaped formats in the context of a short-range, optical fibre link that incorporates low-cost, integrated, high LW $(>500 \mathrm{kHz})$ lasers and report substantial performance gains when using the PCAWGN shaped constellations. We also demonstrate that the presented constellations, shaped with a low-complexity PCAWGN model, incur a minimal performance penalty (versus the theoretically optimum PCAWGN model) when calculating the LLRs of the received signal.

The remainder of this paper is organised as follows: Sec. II describes the system model used in this study. Subsequently, in Sec. III, we introduce the exact PCAWGN channel (i.e., the theoretically optimum model) and the low-complexity approximation used to GS and receive the presented constellations. In Sec. IV, we introduce the information theoretic metrics that are used to evaluate the performance of the GS constellations and the tested demappers. In the following sections, we look at the enhanced shaping algorithm, the approach used to validate the AIRs (Sec. V) and evaluate the post-FEC BER performance of the proposed constellations (Sec. VI). Finally, in Sec. VIII, we summarise the general findings of this study and outline 
the potential research directions for the future work.

\section{SySTEM Model}

A typical implementation of a short-range coherent optical fibre link based on coded modulation (CM) strategy is depicted in Fig. 1 (a), whereas the block diagram in Fig. 1 (b) represents an "equivalent" mathematical model of the transmission link at the top. In such system a binary FEC encoder adds redundancy to a randomly generated sequence of uncoded bits B. Subsequently, the coded bits are mapped to a sequence $\mathbf{X}=\left[X_{1}, X_{2}, \ldots, X_{N}\right]$ of $N$ symbols that are picked from a complex constellation $\mathcal{C}=\left\{C_{1}, C_{2}, \ldots, C_{M}\right\}$ of size $M$. In the next stage, a QPSK pilot overhead $(\mathrm{OH})$ is added into the data frame. Then, the signal is pulse-shaped using a rootraised cosine (RRC) filter, ideally converted into the optical domain at the TX and transmitted through $80-120 \mathrm{~km}$ of a standard single mode fibre (SSMF).

On the receiving side, the incoming single channel signal is optically amplified and passed through an optical pass band filter. Then, at the coherent RX, the carrier phase information is recovered by combining the TX and the LO lasers which, in principle, are tuned to approximately the same wavelengths [27]. As a result, the phase (and phase noise) of the incoming signal $\Theta_{\mathrm{TX}}$ and the $\mathrm{LO} \Theta_{\mathrm{LO}}$ are combined into $\Theta_{\mathrm{TX}+\mathrm{LO}}$.

Subsequently, the signal is converted into the digital domain (sampled at 1 sample/symbol, i.e. baudrate) where DSP algorithms compensate for the $\mathrm{CD}$, perform matched RRC filtering and equalize the received data using the QPSK pilot $\mathrm{OH}$ added to the payload symbols at the TX [28], [29].

At this stage, $\Theta_{\mathrm{TX}+\mathrm{LO}}$ (i.e. the received phase) is usually modelled as a "random-walk" process [1] and requires a CPE algorithm at the RX to be compensated [30]. A common strategy to recover the carrier phase in short-reach systems, such as 400ZR [13], is to embed QPSK pilots between the fixed-length blocks of the payload symbols and use the restored phase information from the pilots $\Theta_{\mathrm{PIL}}$ to calculate the phase estimates $\Theta_{\mathrm{RPN}}$ over the entire data frame by applying digital filtering and interpolation [28], [29]. Then, the CPE output $\boldsymbol{Y}=\left[Y_{1}, Y_{2}, \ldots, Y_{N}\right]$ (i.e. the sequence of the received payload symbols), is passed to a SD demapper to calculate the LLRs that are subsequently decoded, using a binary FEC encoder, into the estimated sequence of the received bits $\widehat{\mathbf{B}}$.

In this work we adopt the pilot-aided CPE approach and assume a commonly used pilot insertion rate of $1 / 32$. However, the authors are aware that the optimal pilot rate may vary for several reasons, not least the individual system specifications or filtering methods [28], [29]. Nevertheless, the modulation formats proposed in this manuscript were GS for the particular SNR-LSTP pairs (can also be taken as SNR-RPN). Therefore, they can be combined with any CPE strategy that is adjusted to produce the output with a similar AWGN power and RPN variance. As an aid to understanding, we provide $\mathrm{LW}$ and symbol time product $(\mathrm{LSTP})^{1}$ metrics that were used with

\footnotetext{
${ }^{1}$ Note that we have deliberately chosen a symbol rate-independent metric (LSTP) to allow comparisons with any reference system. Conversion to laser linewidth from LSTP is straightforward by taking the product of the LSTP with the symbol rate.
}

the proposed CPE approach. It should also be noted that the LSTP parameter is calculated for the combined LW of the two identical lasers (TX and LO).

Consider, now, a system operating at the symbol rate, in which a discrete filter output of the carrier phase recovery (at symbol number $k$ ) is given by

$$
\Theta_{\mathrm{RPN}}[k]=\Theta_{\mathrm{TX}+\mathrm{LO}}[k]-\Theta_{\mathrm{PIL}}[k],
$$

and, as per our previous work in [24, Sec. II], $\Theta_{\text {RPN }}$ is approximated as a random zero-mean Gaussian variable with a variance

$$
\sigma_{\mathrm{RPN}}^{2}=2 \pi \Delta \nu T_{s} .
$$

The term $T_{s}$ denotes the symbol period, and $\Delta \nu$ stands for the combined linewidth of the TX and LO lasers.

As a result, we obtain a discrete-time, memoryless PCAWGN channel, given by

$$
Y[k]=X[k] \cdot e^{j \Theta_{\mathrm{RPN}}[k]}+\mathcal{N}_{0}[k], \text { for } k=1,2, \ldots, N,
$$

where $\mathcal{N}_{0}[k]$ is a zero-mean complex Gaussian random variable that stands for the approximated noise component generated by all other impairments present in the channel (i.e., AWGN).

\section{PCAWGN CHANNEL MODEL}

The theoretical PCAWGN channel law can be computed using the statistical properties of both RPN and AWGN. Generally, the RPN is described as a zero-mean Tikhonov (or a wrapped Gaussian) PDF [5] with a variance $\sigma_{p}^{2}$, whereas the amplitude follows a zero-mean Rician distribution [31]. By combining the two PDFs, we obtain the theoretical PCAWGN channel (i.e., the exact model) as shown in [4, Sec. II].

In practice, implementation of the exact PCAWGN model in hardware is not trivial and requires abundant computational resources. The main reason is the requirement of calculating the modified Bessel functions for each symbol. Hence, it is more economical to compute the LLRs at the receiver with an accurate approximation of the exact PCAWGN model.

In the remainder of this paper, we consider the approximated PCAWGN model from [4, Eq. 17] to geometrically shape the presented constellations. It is expressed as

$$
\begin{aligned}
p(y \mid x) & \approx \frac{K}{\pi \gamma N_{0}} \exp \left(-\frac{\Delta \rho^{2}+(\gamma \Delta \theta)^{2}}{N_{0}}\right), \\
\Delta \rho & =\rho-|x|, \\
\gamma & =\left(\frac{1}{|x| \rho}+\frac{2}{\alpha N_{0}}\right)^{-\frac{1}{2}},
\end{aligned}
$$

where $|\cdot|$ is the absolute value, $\rho=|y|$ and $x$ and $y$ are the values taken by the transmitted and received complex symbols, respectively. The term $N_{0}$ is a variance of an independent, zero-mean, complex random Gaussian variable, $\alpha=\left(\sigma_{p}^{2}\right)^{-1}$ and $\Delta \theta$ is the angular modulo- $\pi$ difference between $x$ and $y$

$$
\Delta \theta=\arctan \left(\frac{\Im\left\{y x^{-1}\right\}}{\Re\left\{y x^{-1}\right\}}\right), \quad \Delta \theta \in[-\pi, \pi) .
$$




\section{INFORMATION THEORETIC METRICS}

To evaluate the BW AIR penalties of the mismatched demappers (auxiliary channel laws), we compared them against the exact channel demapper from [6, Eq. 6]. We describe the difference between the exact and an auxiliary channel law as the information loss $\Delta \mathrm{AIR}_{\mathrm{BW}}$, given by

$$
\Delta \mathrm{AIR}_{\mathrm{BW}}=I_{\mathrm{Ex}}-I_{\mathrm{Aux}},
$$

where $I_{\mathrm{Ex}}$, i.e., channel's GMI [25, Eq. 13], is obtained as

$I_{\mathrm{Ex}} \triangleq \sum_{i=1}^{m} I\left(B_{i} ; Y\right)=\max _{s_{1} \geq 0} \sum_{i=1}^{m} \mathbb{E}_{B_{i}, Y}\left[\log _{2} \frac{f_{Y \mid B_{i}}\left(Y \mid B_{i}\right)^{s_{1}}}{f_{Y}(Y)^{s_{1}}}\right]$

and $I_{\mathrm{Aux}}$, an AIR for a binary FEC scheme using a mismatched decoding metric, is expressed as

$I_{\text {Aux }} \triangleq \sum_{i=1}^{m} I\left(B_{i} ; Y\right)=\max _{s_{2} \geq 0} \sum_{i=1}^{m} \mathbb{E}_{B_{i}, Y}\left[\log _{2} \frac{g_{Y \mid B_{i}}\left(Y \mid B_{i}\right)^{s_{2}}}{g_{Y}(Y)^{s_{2}}}\right]$

The term $\mathbb{E}$ is the expectation, $f_{Y \mid B_{i}}$ denotes the exact channel law from the [6, Eq. 6] and $g_{Y \mid B_{i}}$ is the mismatched channel law from Eq. (4), where $B_{i}$ and $Y$ are distributed according to the exact channel law given by [6, Eq. 6].
While the calculated LLRs in Eqs. (9) and (10) can be scaled, we observed that introducing this additional degree of freedom (i.e., the terms $s_{1}$ and $s_{2}$ ) has a negligible benefit in the context of the presented system specifications. Thus, in the remainder of this work we assumed $s_{1}=1$ and $s_{2}=1$. Moreover, as the CPE strategy used in this paper is shapeagnostic [8], we used 64QAM constellations with Gray bit-tosymbol mappings for the following validation.

\section{CONSTELlation Optimization}

Although it is possible to optimise a constellation for a specific FEC scheme, using AIRs, such as the GMI or mutual information, requires a lot less computational resources to estimate the post-FEC BER performance for a substantial number of good FEC codes [25], [26]. However, in the case of the PCAWGN channel, calculation of the exact channel law requires significant computational resources to implement the Bessel functions. To avoid these bottlenecks, and provide a joint modulation and demodulation strategy, we used the model from the Eq. (4) to maximize the mismatched BW AIR performance (discussed in Sec. IV) of the GS constellations presented [33].
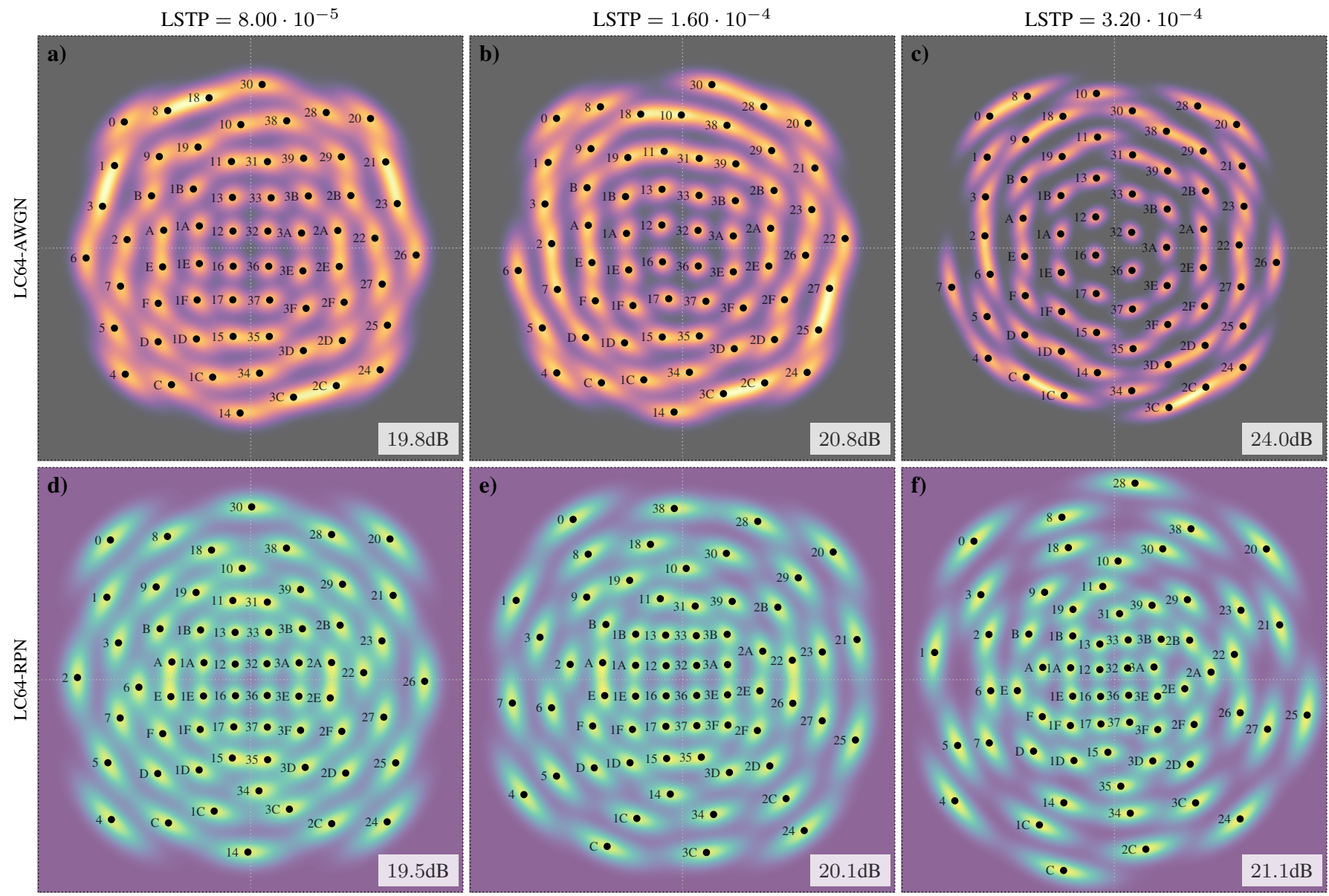

Fig. 2. The RPN shaped (LCM-RPN) 64-ary constellations (per column) for the LSTPs of: a) $8.00 \cdot 10^{-5}$, c) $1.60 \cdot 10^{-4}$ and e) $3.20 \cdot 10^{-4}$. Constellations b), d) and f) are the LCM-AWGN counterparts for the LSTPs of $8.00 \cdot 10^{-5}, 1.60 \cdot 10^{-4}$ and $3.20 \cdot 10^{-4}$, respectively. All the formats depict the optimal symbol arrangement at the $\mathrm{AIR}_{\mathrm{BW}}$ threshold of $0.9 \times \mathrm{m}$ bit/symbol (LDPC), bit-to-symbol mapping is given as hexadecimal numbers. The full dataset of the presented constellations (together with bit-to-symbol mappings) is publicly available at [32]. 


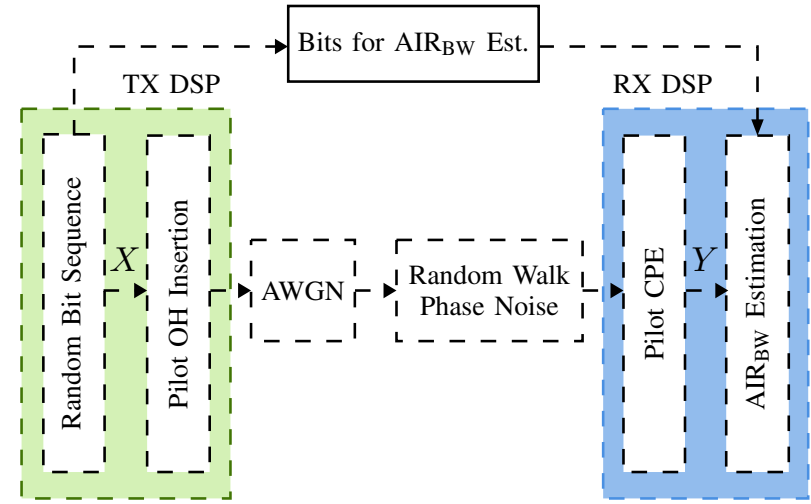

Fig. 3. System model for verification.

\section{A. Optimisation algorithm}

The presented optimisation strategy aims to maximise the mismatched BW AIR using the Eq. (10), of the $M$-ary constellation. The shaping algorithm used in this paper combines the first stage of gradient descent approach from [34] with a bit-to-symbol mapping technique demonstrated in [7].

In contrast to our previous work [24], we replaced the Euclidean distance metric in the optimiser with the proposed PCAWGN approximation from the Eq. (4). Then, to estimate the BW AIR performance for each of the symbol arrangements, we calculated the 3-dimensional Gauss-Hermite integral (with $L=7$ per dimension [35]). As in [24], we modelled the RPN variances (i.e., $\alpha$ values) in the optimiser (via Monte Carlo estimation with $2^{19}$ symbols) to produce approximately the same variance as if the CPE strategy from Sec. V-B was used. Specifically, for each of the SNR-LSTP pair, we measured the average RPN after the CPE and used it in the optimiser to shape the constellation. In a more practical scenario, $\alpha$ could be estimated with, for example, a maximum likelihood algorithm [36, Eq. 4.2]. For the AWGN channel, which herein serves as a reference for the PCAWGN shaped constellations, the Gauss Hermite calculation was reduced to two dimensions representing the real and imaginary parts of the complex AWGN component.

In the remainder of this paper the PCAWGN-optimised formats are referred as "-RPN", whereas the constellations shaped for a purely AWGN channel are referred as "-AWGN". It is also important to emphasise that the following optimisation strategy is not guaranteed to find the global optimum. However, the presented constellations have shown a notable performance advantage over the corresponding QAM formats.

\section{B. Performance validation}

To confirm our assumptions of the statistical model of PCAWGN channel, we validated the GS constellations in a system depicted in Fig. 3. In terms of BW AIR, the system is equivalent to the model from Fig. 1b discussed in Sec. II. In this analysis, we assume that the equalization is ideal and $\mathrm{CD}$ does not have a significant impact on the features of the phase noise. Hence, we focus only on the phase noise after $\mathrm{CPE}$ neglecting the impact of $\mathrm{CD}$. In the presence of $\mathrm{CD}$ an additional equalization enhanced phase noise penalty will be incurred whereby angular laser phase noise is converted into amplitude noise [37]. Such an effect is dependent on symbol rate, fiber type and transmission distance, and is thus beyond the scope of this investigation, but noted here for completeness.

Throughout the validation process, the BW AIRs of the optimised formats via Monte-Carlo simulation, where $2^{21}$ randomly generated symbols (per each tested constellation) were sent through a channel that is impaired by both AWGN and a random-walk phase noise. The optimized constellations were validated using the model in Fig. 3, where the CPE was undertaken with a format-agnostic strategy where QPSK pilots are embedded between every block of 32 data symbols [28], [29]. At the receiver, the pilot phase estimates are interpolated over the entire data frame using a Wiener filter with the coefficients calculated as per [30]. The interpolation correctness is ensured by setting the minimum filter length to 65 taps. Thus, taking at least two consecutive pilot symbols to calculate the phase estimates of the payload. At this point, we expect the filter output to be equivalent to the channel modelled by Eq. [6, Eq. 6], i.e., the exact PCAWGN model. Subsequent to BW AIR estimation, we validated the post-FEC BER performance of the constellations. As a representative example of a common FEC code, we used the 9/10 rate LDPC code from Digital Video Broadcasting standard with a block length of 6480 (i.e., a single LDPC encoded block consists of 58320 data bits and 6480 parity bits) [38].

To ensure a fair comparison, we contrasted the BW AIRs and the post-FEC BERs of the presented constellations with the corresponding AWGN optimised and $M$-QAM (Gray bitmapped) formats. Moreover, for a better reference in terms of achievable shaping gains, we also compared the low complexity channel model (i.e., the PCAWGN approximation) Eq. (4) with a circular Gaussian receiver model [23].

It should be noted that the constellations validated with the reduced complexity PCAWGN model proposed in this paper are referred to as LCM, whereas the EX $M$ denotes the constellations validated with the exact model. Moreover, the corresponding formats validated with the Euclidean distance (ED) receiver are referred to as $\mathrm{ED} M$. Lastly, the entire constellation dataset presented in this paper (i.e., 8 to 64-ary optimisation for purely AWGN and PCAWGN channels) is available to download at [32].

It is also worth noting that the results presented herein should be considered as a conservative estimate of the overall CPE performance. In principle, the proposed CPE algorithm could potentially be enhanced by, for example, pilot rate adjustments and/or incorporating blind phase tracking between the payload symbols. The purpose of these results is to demonstrate the efficacy of the LLR calculation and constellation shaping; as noted previously, the results can be interpreted via RPN variance, which is independent of CPE algorithm and constellation.

Note that the QPSK pilots were set to be equal to the average signal power. Furthermore the reported BW AIRs were multiplied with $31 / 32$ to calculate the net information rate. 
TABLE I

LC64-RPN POST-FEC SHAPING GAINS VS OTHER MODULATION FORMATS

\begin{tabular}{lcccccccc}
\hline LSTP & ED64-AWGN & EX64-AWGN & LC64-AWGN & ED64-QAM & EX64-QAM & LC64-QAM & ED64-RPN & EX64-RPN \\
\hline $8.00 \times 10^{-5}$ & $0.73 \mathrm{~dB}$ & $0.24 \mathrm{~dB}$ & $0.23 \mathrm{~dB}$ & $1.22 \mathrm{~dB}$ & $0.71 \mathrm{~dB}$ & $0.73 \mathrm{~dB}$ & $0.35 \mathrm{~dB}$ & $-0.01 \mathrm{~dB}$ \\
$1.60 \times 10^{-4}$ & $1.97 \mathrm{~dB}$ & $0.70 \mathrm{~dB}$ & $0.69 \mathrm{~dB}$ & $2.65 \mathrm{~dB}$ & $1.13 \mathrm{~dB}$ & $1.14 \mathrm{~dB}$ & $0.60 \mathrm{~dB}$ & $-0.01 \mathrm{~dB}$ \\
$3.20 \times 10^{-4}$ & - & $2.19 \mathrm{~dB}$ & $2.20 \mathrm{~dB}$ & - & $2.59 \mathrm{~dB}$ & $2.59 \mathrm{~dB}$ & - & $-0.02 \mathrm{~dB}$ \\
\hline
\end{tabular}

\section{RESULTS}

In this section, we present the GS results obtained with the algorithm from Section V. To enhance readability of the paper, we will focus on 64-ary constellations. However, the corresponding shaping gains vs ED $M$-QAM formats and gaps to AWGN of the remaining modulation orders of 8,16 and 32 are provided in the Appendix as Figs. 6, 7 and 8, respectively.

\section{A. Optimised 64-ary constellations}

Fig. 2 depicts 64 point constellations optimized at the BW AIR threshold of $0.9 \mathrm{~m} \mathrm{bit} / \mathrm{symbol}$ that corresponds to rate 9/10 LDPC code, which we will use, as an example, in postFEC validation. Here, the PCAWGN channel is visualised as a multi-layered background where the received symbol probability is proportional to the colour brightness. Each column corresponds to LSTPs of $8.00 \times 10^{-5}, 1.60 \times 10^{-4}$ and $3.20 \times 10^{-4}$, respectively.

\section{B. BW AIR performances}

After optimisation, we validated the BW AIRs of the presented 64-ary formats using the low-complexity, exact and
Euclidean demappers. Then, in Sec. VII, we looked at the postFEC BERs for under the assumption of 9/10 LDPC scheme.

As shown in Fig. 4(a) (per column), for all LSTP cases the PCAWGN shaped formats, paired with either the exact PCAWGN (dotted green line) or the proposed (solid green line) model, are clearly better than the corresponding pure AWGN optimised and QAM constellations.

Overall, the RPN shaped constellation have demonstrated significant BW AIR performance advantage over the corresponding AWGN and QAM formats. In the case of LC64RPN, the SNR gains over LC64-AWGN, at the normalised BW AIR threshold of $0.9 \mathrm{~m}$ bit/symbol, ranges from $0.36 \mathrm{~dB}$ at the LSTP of $8.00 \times 10^{-5}$ to $2.65 \mathrm{~dB}$ at the LSTP of $3.20 \times 10^{-4}$ (Fig. 4(c)). Moreover, when compared to the corresponding EX64-QAM and EX64-AWGN formats, the LC64-RPN is $3.12 \mathrm{~dB}$ and $2.67 \mathrm{~dB}$ better.

To evaluate the impact of using the low-complexity PCAWGN channel model on the LLR calculation, we also compared the LC64 demapped constellations with the corresponding formats received with the Euclidean distance demapper (denoted as $\operatorname{ED} M$ ). The resulting gains, with respect to ED64-QAM, are shown in Fig. 4(d). It can be observed that the enhanced receiver model alone significantly improves the per-
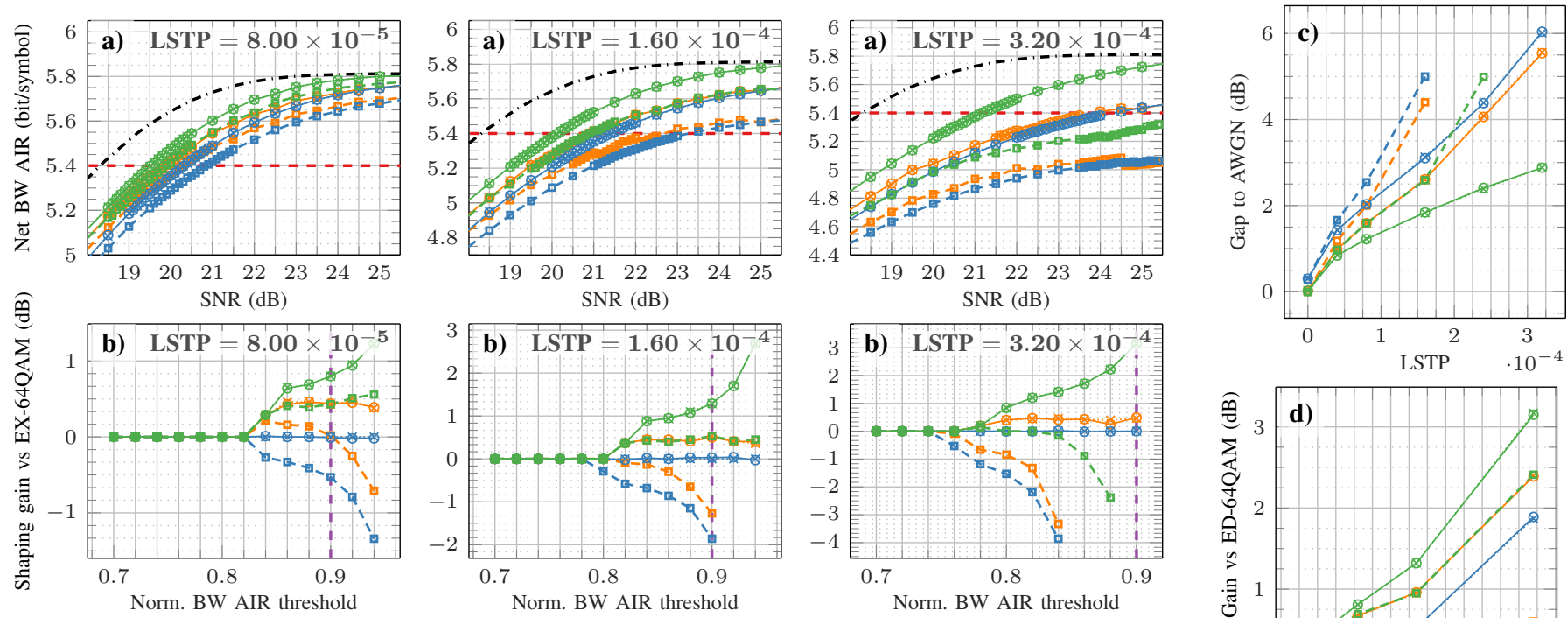

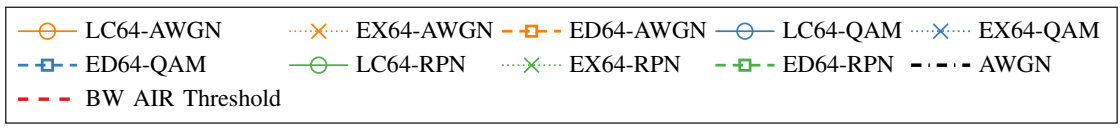

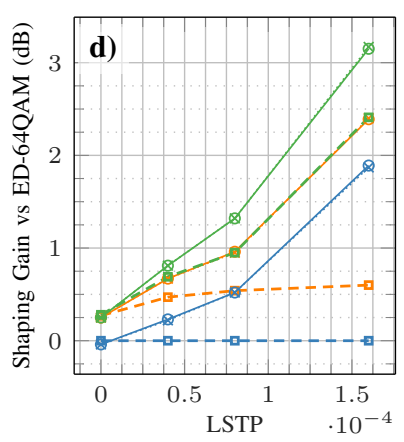

Fig. 4. BW AIR performance curves of 64-ary constellations at LSTPs of $8.00 \cdot 10^{-5}, 1.60 \cdot 10^{-4}$ and $3.20 \cdot 10^{-4}$. The first three columns stand for: (a) BW AIR performance curves, (b) shaping gains in the required SNR over a range of normalised BW AIR thresholds (versus EX64-QAM). The last column represents SNR gaps to AWGN channel performance of AWGN optimised formats (c), and shaping gains in the required SNR over the corresponding ED-64QAM formats (d). 

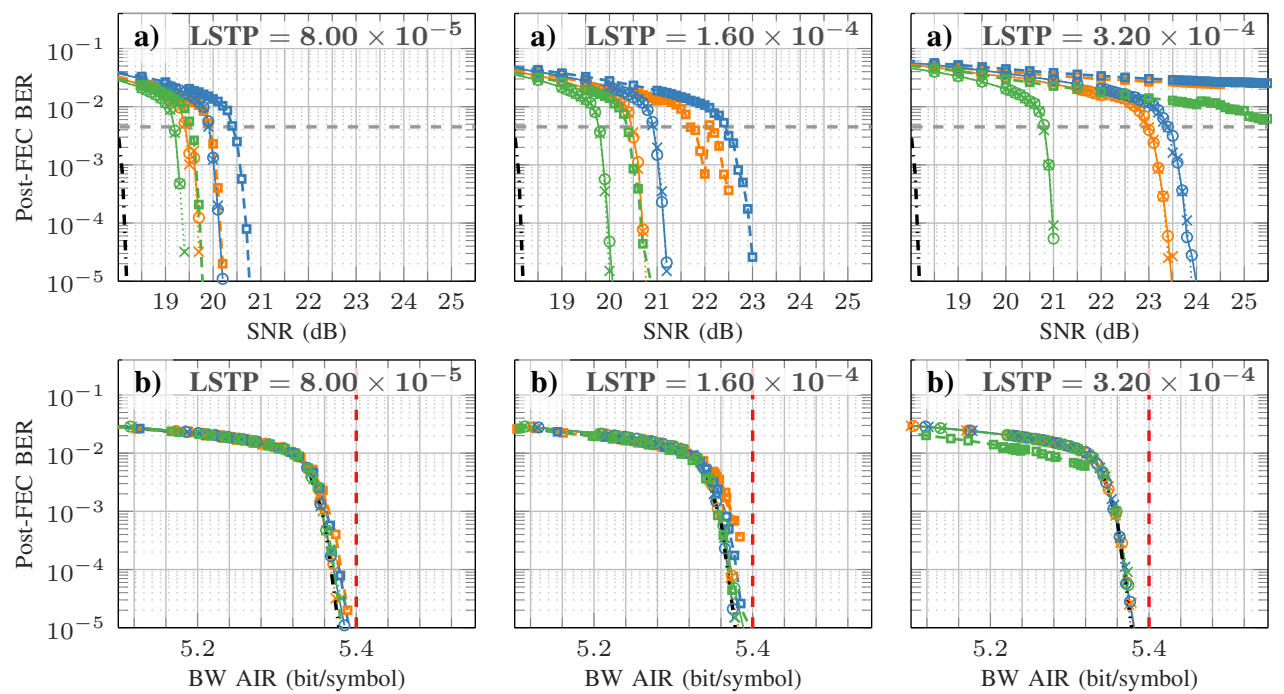

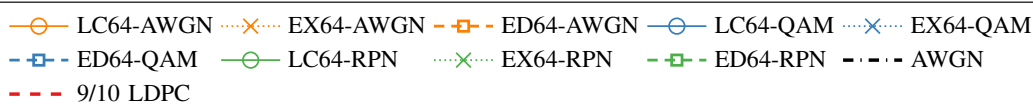
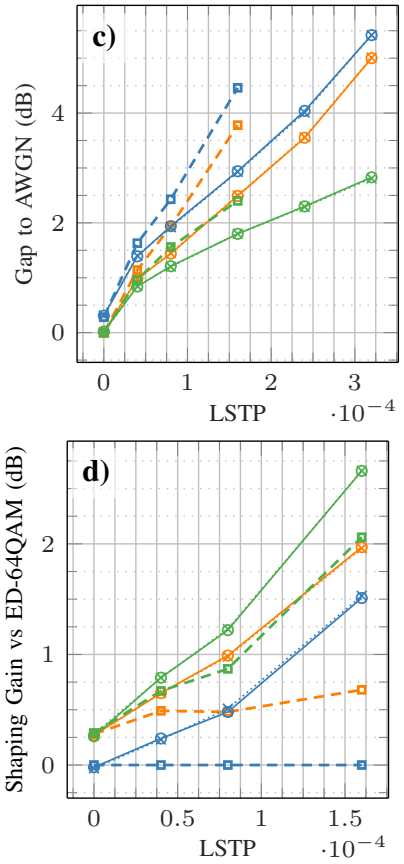

Fig. 5. Post-FEC performances of the 64-ary constellations with (a) 9/10 rate LDPC scheme and (b) Post-FEC BER to BW AIR relationship for the LSTPs of $8.00 \cdot 10^{-5}, 1.60 \cdot 10^{-4}$ and $3.20 \cdot 10^{-4}$ (per column). The last column represents post-FEC SNR gaps to pure AWGN channel performance of AWGN optimised format (c), and shaping gains in the required SNR over the corresponding ED-64QAM formats (d).

formance of all the compared formats and, for all 3 modulation formats, allows the system to reach the BW AIR threshold at the LSTP of $3.2 \times 10^{-4}$. Moreover, in all of the demonstrated cases, the exact model (i.e., EX64- formats) maintains a negligible performance advantage (that is, $<0.02 \mathrm{~dB}$ ) over the proposed approximation. Hence, there is no benefit of using the theoretically optimum, but computationally complex, model over the proposed low-complexity approximation.

Furthermore, to provide a fairer overview of the potential impact of PCAWGN shaping for other code rates, we also estimated the shaping gains over a wider range of the normalised BW AIR thresholds. As shown in Fig. 4(b), where we compared the shaping gains of the presented constellations versus EX64-QAM, it turns out that for 64-ary formats the shaping gains increase proportionally to the investigated code rates. This could be justified with the decreasing impact of the circular AWGN in high SNR regions, which effectively makes the RPN a dominant source of the channel impairments.

\section{SD LDPC FEC RESULTS}

Subsequent to BW AIR validation, we looked at post-FEC BERs of the presented formats using LDPC scheme with 9/10 rate. The resulting performances for the LSTP values of: $8.00 \times 10^{-5}, 1.60 \times 10^{-4}$ and $3.20 \times 10^{-4}$ (per column) are shown in Fig. 5(a). Under the assumption of error-free transmission at $4.5 \times 10^{-3}$ post-FEC BER, provided by, for example, an outer hard decision staircase code [39], we can see that all the transmitted constellations achieve error-free performance (post-FEC BER below $10^{-15}$, see [39, Fig. 8]) at the estimated BW AIR threshold of 5.4 bits/symbol, as shown in Fig. 5(b).
We also looked at the resulting gaps to AWGN optimised constellation in purely AWGN channel (Fig. 5 (c)), and the corresponding shaping gains over ED64-QAM (Fig. 5 (d)). Similar to the results from Sec. VI-B, the post-FEC shaping gains over LC64-AWGN, for the LSTPs of $8.00 \times 10^{-5}$, $1.60 \times 10^{-4}$ and $3.20 \times 10^{-4}$ span from $0.23 \mathrm{~dB}$ to $2.19 \mathrm{~dB}$. Moreover, when compared to LC64-QAM formats, the proposed constellations perform up to $2.59 \mathrm{~dB}$ better by means of post-FEC BER.

Lastly, as shown in the last column of Tab. I, in all of the demonstrated cases, the post-FEC BERs for the exact model (i.e., EX64- formats) maintain a negligible performance advantage over the proposed (LC64- formats) approximation $(<0.02 \mathrm{~dB})$.

\section{CONCLUSION}

In this work we have reviewed the existing literature on modelling and LLR calculation for PCAWGN channels, a channel representative of a coherent optical transmission system with high levels of phase noise. We have used a lowcomplexity PCAWGN approximation that approaches optimal performance over a wide range of SNR and phase noise values to develop GS constellations optimised for the PCAWGN channel and for the low-complexity receiver metric.

In the high SNR region, the proposed shaped 64-ary constellations showed up to $3.12 \mathrm{~dB}$ BW AIR gains over the conventional modulation formats. Moreover, we showed that at a target $9 / 10$ rate shaping gains translated exactly to postFEC BER gains when using an SD LDPC code. The most significant shaping gains, up to $2.59 \mathrm{~dB}$ and $2.19 \mathrm{~dB}$ over the respective PR64-QAM and PR64-AWGN formats, resulted 
TABLE II

POST-FEC SHAPING GAINS FOR LC8-RPN, LC16-RPN AND LC32-RPN VS OTHER MODULATION FORMATS

\begin{tabular}{|c|c|c|c|c|c|c|c|c|}
\hline LSTP / LC8-RPN vs & ED8-AWGN & EX8-AWGN & LC8-AWGN & ED8-QAM & EX8-QAM & LC8-QAM & ED8-RPN & EX8-RPN \\
\hline $8.00 \times 10^{-5}$ & $0.12 \mathrm{~dB}$ & $0.09 \mathrm{~dB}$ & $0.12 \mathrm{~dB}$ & $0.89 \mathrm{~dB}$ & $0.80 \mathrm{~dB}$ & $0.81 \mathrm{~dB}$ & $0.00 \mathrm{~dB}$ & $-0.02 \mathrm{~dB}$ \\
\hline $1.60 \times 10^{-4}$ & $0.39 \mathrm{~dB}$ & $0.34 \mathrm{~dB}$ & $0.37 \mathrm{~dB}$ & $1.11 \mathrm{~dB}$ & $0.96 \mathrm{~dB}$ & $0.96 \mathrm{~dB}$ & $0.05 \mathrm{~dB}$ & $-0.03 \mathrm{~dB}$ \\
\hline $3.20 \times 10^{-4}$ & $0.92 \mathrm{~dB}$ & $0.79 \mathrm{~dB}$ & $0.80 \mathrm{~dB}$ & $1.54 \mathrm{~dB}$ & $1.16 \mathrm{~dB}$ & $1.17 \mathrm{~dB}$ & $0.14 \mathrm{~dB}$ & $-0.03 \mathrm{~dB}$ \\
\hline LSTP / LC16-RPN vs & ED16-AWGN & EX16-AWGN & LC16-AWGN & ED16-QAM & EX16-QAM & LC16-QAM & ED16-RPN & EX16-RPN \\
\hline $8.00 \times 10^{-5}$ & $0.25 \mathrm{~dB}$ & $0.14 \mathrm{~dB}$ & $0.15 \mathrm{~dB}$ & $0.31 \mathrm{~dB}$ & $0.18 \mathrm{~dB}$ & $0.19 \mathrm{~dB}$ & $0.06 \mathrm{~dB}$ & $-0.01 \mathrm{~dB}$ \\
\hline $1.60 \times 10^{-4}$ & $0.48 \mathrm{~dB}$ & $0.25 \mathrm{~dB}$ & $0.28 \mathrm{~dB}$ & $0.58 \mathrm{~dB}$ & $0.31 \mathrm{~dB}$ & $0.32 \mathrm{~dB}$ & $0.14 \mathrm{~dB}$ & $-0.01 \mathrm{~dB}$ \\
\hline $8.00 \times 10^{-5}$ & $0.48 \mathrm{~dB}$ & $0.26 \mathrm{~dB}$ & $0.26 \mathrm{~dB}$ & $0.69 \mathrm{~dB}$ & $0.48 \mathrm{~dB}$ & $0.48 \mathrm{~dB}$ & $0.15 \mathrm{~dB}$ & $-0.01 \mathrm{~dB}$ \\
\hline $1.60 \times 10^{-4}$ & $0.98 \mathrm{~dB}$ & $0.46 \mathrm{~dB}$ & $0.48 \mathrm{~dB}$ & $1.21 \mathrm{~dB}$ & $0.68 \mathrm{~dB}$ & $0.69 \mathrm{~dB}$ & $0.30 \mathrm{~dB}$ & $-0.01 \mathrm{~dB}$ \\
\hline $3.20 \times 10^{-4}$ & $2.95 \mathrm{~dB}$ & $0.98 \mathrm{~dB}$ & $1.00 \mathrm{~dB}$ & $3.16 \mathrm{~dB}$ & $1.21 \mathrm{~dB}$ & $1.20 \mathrm{~dB}$ & $0.96 \mathrm{~dB}$ & $-0.01 \mathrm{~dB}$ \\
\hline
\end{tabular}

from bringing the geometric shaping and the proposed lowcomplexity demapper together.

We also showed that in the high SNR regime the lowcomplexity approximation incurs a negligible performance loss versus the exact PCAWGN metric. In the case of 64-ary formats, the RPN shaped constellations received with the lowcomplexity demapper experienced penalties $<0.02 \mathrm{~dB}$ versus their counterparts received with the exact metric. Hence, the low-complexity PCAWGN approximation not only requires lower computational resources than the exact PCAWGN metric but also provides a close to theoretically optimal performance. Thus, we believe that it can be considered a highly efficient PCAWGN approximation to be implemented (jointly with geometric shaping) in short-range, partially coherent schemes based on high linewidth lasers. Such systems include 400ZR or short-reach passive optical networks.

In summary, we anticipate that geometric constellation shaping, in conjunction with a more accurate, low-complexity demapper, could be used either as a tool to relax linewidth and/or CPE requirements, or to simply increase SNR tolerance in the presence of RPN.

\section{APPENDIX}

The shaping gains over the corresponding $M$-QAM formats and gaps to purely AWGN channel for the modulation orders of $M=8,16$ and 32, supported by Tab. II.
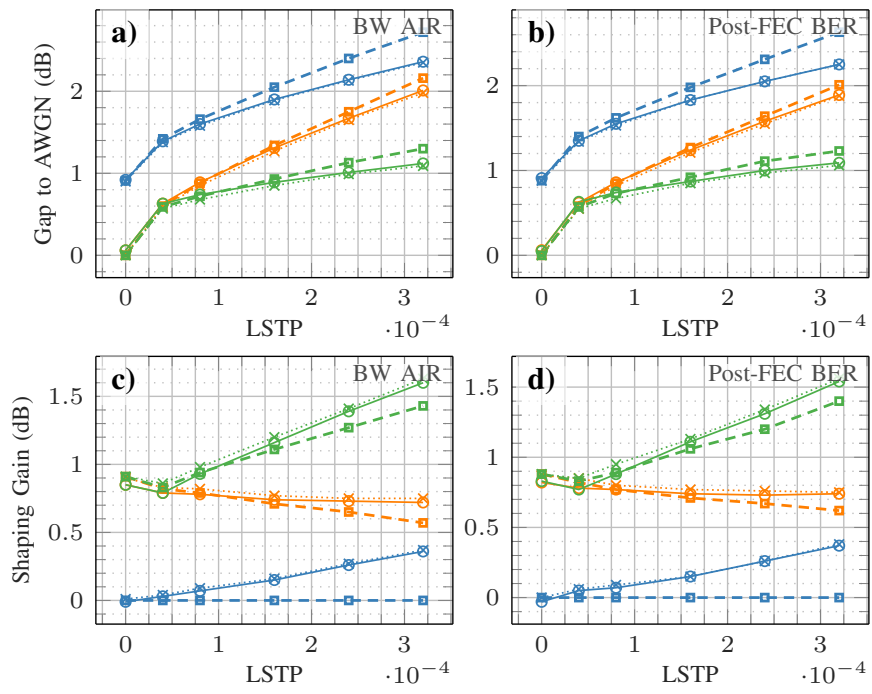

$$
\begin{aligned}
& \text { - } \mathrm{LC} 8 \text {-AWGN ……... EX8-AWGN - - - ED8-AWGN } \\
& \text { ○ LC8-QAM ……. EX8-QAM - }- \text { - ED8-QAM } \\
& \text { ○-LC8-RPN …… EX8-RPN - } \square-- \text { ED8-RPN }
\end{aligned}
$$

Fig. 6. SNR gaps to AWGN channel performance of AWGN optimised formats (a-b), and shaping gains in the required SNR over the corresponding ED-8QAM formats (c-d).

\section{ACKNOWLEDGMENTS}

The authors wish to extend their thanks to Prof Per LarssonEdefors and Erik Börjeson from Chalmers University for enlightening discussions, particularly on the topic of the hardware implementation of the PCAWGN models. 

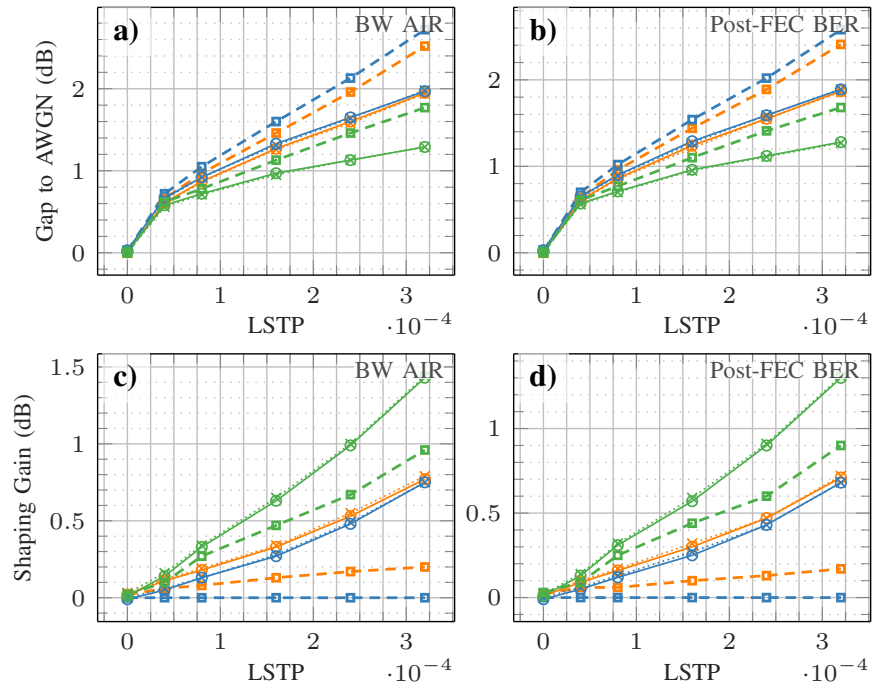

\begin{tabular}{|c|c|c|}
\hline- LC16-AWGN & $\times$ EX16-AWGN & - ㅁ- - ED16-AWGN \\
\hline - LC16-QAM & $\times$ EX16-QAM & - घ- - ED16-QAM \\
\hline$-\mathrm{LC} 16-\mathrm{RPN}$ & $\times$ EX16-RPN & - \\
\hline
\end{tabular}

Fig. 7. SNR gaps to AWGN channel performance of AWGN optimised formats (a-b), and shaping gains in the required SNR over the corresponding ED-16QAM formats (c-d).
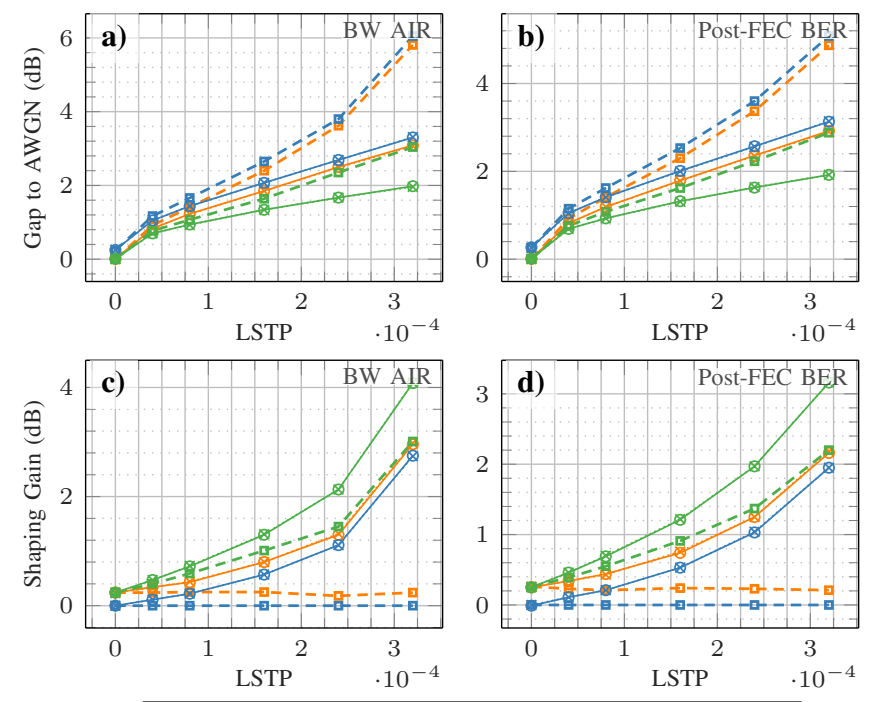

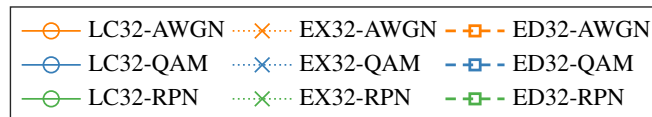

Fig. 8. SNR gaps to AWGN channel performance of AWGN optimised formats $(a-b)$, and shaping gains in the required SNR over the corresponding ED-32QAM formats (c-d).

\section{REFERENCES}

[1] W. T. Silfvast, Laser fundamentals. Cambridge University Press, 2009.

[2] S. J. Savory, "Digital coherent optical receivers: Algorithms and subsystems," IEEE Journal on Selected Topics in Quantum Electronics, vol. 16, no. 5, pp. 1164-1179, 2010.

[3] G. J. Foschini, R. D. Gitlin, and S. B. Weinstein, "On the selection of a two-dimensional signal constellation in the presence of phase jitter and gaussian noise,' The Bell System Technical Journal, vol. 52, no. 6, pp. 927-965, 1973.

[4] M. Sales-Llopis and S. J. Savory, "Approximating the Partially Coherent Additive White Gaussian Noise Channel in Polar Coordinates," IEEE Photonics Technology Letters, vol. 31, no. 11, pp. 833-836, 2019.

[5] B. Goebel, R. Essiambre, G. Kramer, P. J. Winzer, and N. Hanik, "Calculation of Mutual Information for Partially Coherent Gaussian
Channels With Applications to Fiber Optics," IEEE Transactions on Information Theory, vol. 57, no. 9, pp. 5720-5736, 2011.

[6] F. Kayhan and G. Montorsi, "Constellation Design for Memoryless Phase Noise Channels," IEEE Transactions on Wireless Communications, vol. 13, no. 5, pp. 2874-2883, 2014.

[7] B. Chen, C. Okonkwo, H. Hafermann, and A. Alvarado, "Increasing Achievable Information Rates via Geometric Shaping," in Proc. of European Conference on Optical Communication (ECOC), Paper We1F.4, Sep. 2018, pp. 1-3.

[8] T. Gerard, H. Dzieciol, E. Sillekens, Y. Wakayama, A. Alvarado, R. I. Killey, P. Bayvel, and D. Lavery, "Coded Modulation for 100G Coherent EPON," Journal of Lightwave Technology, vol. 38, no. 3, pp. 564-572, 2020.

[9] E. Agrell, "Database of sphere packings." [Online]. Available: https://codes.se/packings/

[10] G. Forney, R. Gallager, G. Lang, F. Longstaff, and S. Qureshi, "Efficient Modulation for Band-Limited Channels," IEEE Journal on Selected Areas in Communications, vol. 2, no. 5, pp. 632-647, Sep. 1984.

[11] B. W. Kernighan and S. Lin, "Heuristic solution of a signal design optimization problem," The Bell System Technical Journal, vol. 52, no. 7, pp. 1145-1159, Sep. 1973.

[12] G. Foschini, R. Gitlin, and S. Weinstein, "Optimization of TwoDimensional Signal Constellations in the Presence of Gaussian Noise," IEEE Transactions on Communications, vol. 22, no. 1, pp. 28-38, January 1974.

[13] “OIF 400ZR project start (OIF2016.400.04)." [Online]. Available: https://www.oiforum.com/

[14] J. D. Reis, A. Shahpari, R. Ferreira, S. Ziaie, D. M. Neves, M. Lima, and A. L. Teixeira, "Terabit+ $(192 \times 10 \mathrm{~Gb} / \mathrm{s})$ Nyquist Shaped UDWDM Coherent PON With Upstream and Downstream Over a $12.8 \mathrm{~nm}$ Band," Journal of Lightwave Technology, vol. 32, no. 4, pp. 729-735, 2014.

[15] R. Krishnan, A. Graell i Amat, T. Eriksson, and G. Colavolpe, "Constellation Optimization in the Presence of Strong Phase Noise," IEEE Transactions on Communications, vol. 61, no. 12, pp. 5056-5066, 2013.

[16] T. Pfau, X. Liu, and S. Chandrasekhar, "Optimization of 16-ary Quadrature Amplitude Modulation constellations for phase noise impaired channels," in 2011 37th European Conference and Exhibition on Optical Communication, 2011, pp. 1-3.

[17] S. Kirkpatrick, C. D. Gelatt, and M. P. Vecchi, "Optimization by Simulated Annealing," Science, vol. 220, no. 4598, pp. 671-680, 1983.

[18] G. Liga, A. Alvarado, E. Agrell, and P. Bayvel, "Information rates of next-generation long-haul optical fiber systems using coded modulation," Journal of Lightwave Technology, vol. 35, no. 1, pp. 113-123, 2017.

[19] B. Kwak, N. Song, B. Park, and D. S. Kwon, "Spiral QAM: A Novel Modulation Scheme Robust in the Presence of Phase Noise," in 2008 IEEE 68th Vehicular Technology Conference, 2008, pp. 1-5.

[20] A. Ugolini, A. Piemontese, and T. Eriksson, "Spiral Constellations for Phase Noise Channels," IEEE Transactions on Communications, vol. 67, no. 11, pp. 7799-7810, 2019.

[21] D. Pilori, A. Nespola, F. Forghieri, and G. Bosco, "Non-linear phase noise mitigation over systems using constellation shaping," Journal of Lightwave Technology, vol. 37, no. 14, pp. 3475-3482, 2019.

[22] V. Talreja, T. Koike-Akino, Y. Wang, D. S. Millar, K. Kojima, K. Parsons, "End-to-End Deep Learning for Phase Noise-Robust MultiDimensional Geometric Shaping," in Proc. of European Conference on Optical Communications (ECOC), Paper Th1D-4, 2020.

[23] N. Merhav, G. Kaplan, A. Lapidoth, and S. Shamai Shitz, "On information rates for mismatched decoders," IEEE Transactions on Information Theory, vol. 40, no. 6, pp. 1953-1967, 1994.

[24] H. Dzieciol, G. Liga, E. Sillekens, P. Bayvel, and D. Lavery, "Geometric shaping of 2-dimensional constellations in the presence of laser phase noise," Journal of Lightwave Technology, pp. 1-1, 2020.

[25] A. Alvarado, E. Agrell, D. Lavery, R. Maher, and P. Bayvel, "Replacing the Soft-Decision FEC Limit Paradigm in the Design of Optical Communication Systems," Journal of Lightwave Technology, vol. 34, no. 2, pp. 707-721, 2016.

[26] E. Agrell, M. Secondini, S. Superiore, and S. Anna, "InformationTheoretic Tools for Optical Communications Engineers - IEEE Conference Publication," 2018 IEEE Photonics Conference (IPC), pp. 1-5, 2018.

[27] S. J. Savory, "Digital coherent optical receivers: Algorithms and subsystems," IEEE Journal of selected topics in quantum electronics, vol. 16, no. 5, pp. 1164-1179, 2010.

[28] M. Mazur, J. Schröder, A. Lorences-Riesgo, T. Yoshida, M. Karlsson, and P. A. Andrekson, "Overhead-optimization of pilot-based digital signal processing for flexible high spectral efficiency transmission," Opt. Express, vol. 27, no. 17, pp. 24654-24669, Aug 2019. 
[29] Y. Wakayama, E. Sillekens, L. Galdino, D. Lavery, R. Killey, and P. Bayvel, "Increasing achievable information rates with pilot-based DSP in standard intradyne detection," in Proc. of European Conference on Optical Communications (ECOC), Paper W.1.B.5, 2019.

[30] E. Ip and J. M. Kahn, "Feedforward Carrier Recovery for Coherent Optical Communications," Journal of Lightwave Technology, vol. 25, no. 9, pp. 2675-2692, Sep. 2007.

[31] S. O. Rice, "Statistical properties of a sine wave plus random noise," The Bell System Technical Journal, vol. 27, no. 1, pp. 109-157, 1948.

[32] H. Dzieciol, "2D Geometrically Shaped Constellations for a Low Complexity Partially-Coherent AWGN Demapper," 2021. [Online]. Available: https://dx.doi.org/10.21227/y1sh-w048

[33] L. Schmalen, A. Alvarado, and R. Rios-Muller, "Performance Prediction of Nonbinary Forward Error Correction in Optical Transmission Experiments," Journal of Lightwave Technology, vol. 35, no. 4, pp. 1015-1027, 2017.

[34] F. Schreckenbach, N. Gortz, J. Hagenauer, and G. Bauch, "Optimized Symbol Mappings for Bit-Interleaved Coded Modulation with Iterative Decoding," in Proc. of IEEE Global Telecommun. Conf., vol. 6., vol. 6, Dec. 2003, pp. 3316-3320 vol.6.

[35] H. Salzer, R. Zucker, and R. Capuano, "Table of the Zeros and Weight Factors of the First Twenty Hermite Polynomials," Journal of Research of the National Bureau of Standards, vol. 48, no. 2, p. 111, 1952.

[36] M. Sales Llopis, "Optical fibre communication over a noisy partially coherent channel," Ph.D. dissertation, University of Cambridge, 2020.

[37] I. Fatadin and S. J. Savory, "Impact of phase to amplitude noise conversion in coherent optical systems with digital dispersion compensation," Opt. Express, vol. 18, no. 15, pp. 16273-16278, Jul 2010. [Online]. Available: http://www.opticsexpress.org/abstract.cfm?URI=oe18-15-16273

[38] DVBS, "Digital Video Broadcasting (DVB); Second Generation Framing Structure, Channel Coding and Modulation Systems for Broadcasting, Interactive Services, News Gathering and Other Broadband Satellite Applications(DVB-S2)," Eur. Standard ETSI EN 302307 V1.2.1 (200908), vol. 1, Aug. 2009.

[39] B. P. Smith, A. Farhood, A. Hunt, F. R. Kschischang, and J. Lodge, "Staircase Codes: FEC for 100Gb/s OTN," IEEE Journal of Lightwave Technology, vol. 30, no. 1, pp. 110-117, 2012. 